\title{
LINEARLY CONSTRAINED LQ AND LQG OPTIMAL CONTROL
}

\author{
A.E.B. LIM*, J.B. MOORE* and L. FAYBUSOVICH** \\ *Department of Systems Engineering and Cooperative Research Centre for Robust and Adaptive Systems, Research \\ School of Information Sciences and Engineering, Australian National University, Canberra ACT 0200, Australia. \\ **Department of Mathematics, University of Notre Dame, Mail Distribution Center, Notre Dame, IN 46556.
}

\begin{abstract}
It has recently been shown that the logarithmic barrier method for solving finite-dimensional, linearly constrained quadratic optimization problems can be extended to an infinite-dimensional setting with complexity estimates similar to the finite dimensional case. As a consequence, an efficient computational method for solving the linearly constrained LQ control problem is now available. In this paper, we solve the linearly constrained LQG control problem by generalizing the Separation Theorem. We show how the logarithmic barrier method can be used to determine the optimal control for the constrained LQG problem.
\end{abstract}

Key Words. Optimal control; linear constraints; interior-point methods; logarithmic barrier method; LQG control; Separation Theorem.

\section{INTRODUCTION}

The solution of the unconstrained linear quadratic (LQ) optimal control problem is well known (Anderson and Moore, 1989). Several methadologies for solving the linearly constrained case have also been developed (Faybusovich, 1982; Faybusovich and Moore, 1995; Faybusovich and Moore, 1996). Faybusovich and Moore (1995) and Faybusovich and Moore (1996), the interior point methodology (IPM) for solving the quadratic programming problems is extended to the infinite-dimensional setting with complexity estimates similar to the finitedimensional case. When applied to the constrained LQ optimal control problem, in the case of linear or quadratic constraints, the authors show that the optimal control can be obtained by solving a sequence of unconstrained LQ problems together with a sequence of finite dimensional linear algebraic equations. In this paper, our focus is on the stochastic model case and so for simplicity we first derive essentially the same result as in Faybusovich and Moore (1995) for the discrete-time LQ optimal control problem. Next, we solve the linearly constrained LQG optimal control problem by generalizing the Separation Theorem. We show that the IPM result for the constrained LQ problem can be used to solve the constrained LQG problem, with all the advantages of IPM's being true (fast convergence and feasible sub-optimal iterates). In future studies, we explore continuous time versions of this Separation Theorem.
In this paper, we shall consider linear integral constraints of the form (3) for the deterministic problem, and (34) for the stochastic problem. As well as constraints of the form (3) or (34), many constraints which arise in practise can be viewed as special cases of (3) and (34) and include for example, bounds on the state or control (eg, $\left|x_{k}\right| \leq c_{k},\left|u_{k}\right| \leq d_{k}$ ) or bounds on the allowable change in the values of the state or control (eg. $\left|x_{k+1}-x_{k}\right| \leq f_{k}, \mid u_{k+1}-u_{k} \leq g_{k}$ ).

\section{IPM APPROACH TO LQ CONTROL}

The logarithmic barrier method is an iterative interior point optimization method (den Hertog, 1994). In this algorithm, an improved estimate of the optimal solution is obtained by adding a term known as a Newton step to the current estimate. In this section, the recent infinite-dimensional generalization of the logarithmic barrier method (Faybusovich and Moore, 1995) is applied to the discrete-time LQ optimal control problem. We show that the Newton step is calculated by solving an unconstrained LQ optimal control problem together with a finite-dimensional system of linear algebraic equations.

Consider the linear system

$$
x_{k+1}=A_{k} x_{k}+B_{k} u_{k}, \quad x_{0}=\mu
$$


with cost and constraint functionals

$$
\begin{aligned}
f_{0}(x, u) & =\frac{1}{2} \sum_{k=1}^{N}\left(x_{k}^{\prime} Q_{k} x_{k}+u_{k-1}^{\prime} R_{k} u_{k-1}\right) \\
l_{i}(x, u) & =\sum_{k=1}^{N}\left(a_{k}^{(i)}{ }^{\prime} x_{k}+b_{k}^{(i)}{ }^{\prime} u_{k-1}\right) \\
i & =1, \cdots, m_{c}
\end{aligned}
$$

where $x_{k} \in \mathbf{R}^{n}, u_{k} \in \mathbf{R}^{m}, A_{k} \in \mathbf{R}^{n \times n}, B_{k} \in \mathbf{R}^{n \times m}$, $Q_{k} \in \mathbf{R}^{n \times n}, R_{k} \in \mathbf{R}^{m \times m}, a_{k}^{(i)} \in \mathbf{R}^{n}$ and $b_{k}^{(i)} \in$ $\mathbf{R}^{m}$ where $Q_{k}$ is symmetric positive semi-definite and $R_{k}$ is symmetric positive definite. The linearly constrained optimal control task is to find a control $u^{*}=$ $\left(u_{0}, \cdots, u_{N-1}\right), u_{k} \in \mathbf{R}^{m}$ which minimizes the performance index (2) and satisfies the constraints $l_{i}(x, u) \leq$ $c_{i}, i=1, \cdots, m_{c}$.

If $x=\left(x_{1}, \cdots, x_{N}\right)$ and $u=\left(u_{0}, \cdots, u_{N-1}\right)$ satisfy (1), we shall refer to $z=(x, u)$ as a feasible state-control pair. For such $z$, we denote the Newton step, parameterized by $\beta>0$ by

$$
\Delta(z, \beta)=\left[\begin{array}{l}
\xi \\
r
\end{array}\right]
$$

where $\xi=\left(\xi_{1}, \cdots, \xi_{N}\right)$ and $r=\left(r_{0}, \cdots, r_{N-1}\right)$. Also, the sum

$$
z^{(1)}=z+\Delta(z, \beta)
$$

means $z^{(1)}=\left(x^{(1)}, u^{(1)}\right)$ where $x^{(1)}=\left(x_{1}^{(1)}, \cdots, x_{N}^{(1)}\right)$ with $x_{k}^{(1)}=x_{k}+\xi_{k}$ and $u^{(1)}=\left(u_{0}^{(1)}, \cdots, u_{N-1}^{(1)}\right)$ with $u_{k}^{(1)}=u_{k}+r_{k}$. Let $\beta>0$ be fixed, and $z=(x, u)$ be a feasible state-control pair. Define

$$
\begin{aligned}
f(z, \beta) & =\beta f_{0}(z)-\sum_{i=1}^{m_{c}} \ln \left(c_{i}-l_{i}(z)\right) \\
s_{i}(z) & =c_{i}-l_{i}(z)
\end{aligned}
$$

It can be shown that

$$
\nabla f(z, \beta)=\left[\begin{array}{l}
\bar{x} \\
\bar{u}
\end{array}\right]
$$

where $\bar{x}=\left(\bar{x}_{1}, \cdots, \bar{x}_{N}\right), \bar{u}=\left(\bar{u}_{1}, \cdots, \bar{u}_{N}\right)$ with

$$
\begin{aligned}
& \bar{x}_{k}=\beta Q_{k} x_{k}+\sum_{i=1}^{m_{c}} \frac{a_{k}^{(i)}}{s_{i}(z)} \\
& \bar{u}_{k}=\beta R_{k} u_{k-1}+\sum_{i=1}^{m_{c}} \frac{b_{k}^{(i)}}{s_{i}(z)}
\end{aligned}
$$

Let $\xi$ and $r$ be the components of the Newton step (4) and denote

$$
d_{i}=l_{i}(\xi, r)
$$

Define

$$
\gamma(z, \beta) \cdot\left[\begin{array}{l}
\xi \\
r
\end{array}\right]=\left[\begin{array}{l}
v \\
w
\end{array}\right]
$$

where $v=\left(v_{1}, \cdots, v_{N}\right), w=\left(w_{1}, \cdots, w_{N}\right)$ with

$$
\begin{aligned}
v_{k} & =\beta Q_{k} \xi_{k}+\sum_{i=1}^{m_{c}} \frac{d_{i}}{s_{i}^{2}(z)} a_{k}^{(i)} \\
w_{k} & =\beta R_{k} r_{k-1}+\sum_{i=1}^{m_{c}} \frac{d_{i}}{s_{i}^{2}(z)} b_{k}^{(i)}
\end{aligned}
$$

Let the sets $X$ and $X^{\perp}$ be defined by

$$
\begin{aligned}
X & =\left\{\left[\begin{array}{l}
x \\
u
\end{array}\right]: \begin{array}{l}
x_{k+1}=A_{k} x_{k}+B_{k} u_{k} \\
x_{0}=0
\end{array}\right\} \\
X^{\perp} & =\left\{\left[\begin{array}{l}
\bar{p} \\
\bar{q}
\end{array}\right]: \begin{array}{l}
\bar{p}=\left(\bar{p}_{1}, \cdots, \bar{p}_{N}\right) \\
\bar{q}=\left(\bar{q}_{1}, \cdots, \bar{q}_{N}\right)
\end{array}\right\}
\end{aligned}
$$

where

$$
\begin{aligned}
\bar{p}_{k} & =-p_{k-1}+A_{k}^{\prime} p_{k} \\
\bar{q}_{k} & =B_{k-1}^{\prime} p_{k-1}
\end{aligned}
$$

with $p=\left(p_{0}, \cdots, p_{N}\right)$ being any sequence such that $p_{k} \in$ $\mathbf{R}^{n}$ and $p_{N}=0$, and $A_{N}=0$.

It is shown by Faybusovich and Moore (1995) that the Newton step $\Delta(z, \beta)$ is the unique solution of the equation

$$
\Delta(z, \beta)=-\gamma^{-1}(z, \beta)[\nabla f(z, \beta)-\mu(z, \beta)]
$$

where $\mu(y, \beta) \in X^{\perp}$ is the unique vector which must be determined so that $\Delta(z, \beta) \in X$ where $X$ and $X^{\perp}$ are given by $(8)$ and $(9)$ respectively. Therefore, the problem of finding $\Delta(z, \beta)$ is equivalent to the one of finding the unique $\mu(z, \beta) \in X^{\perp}$ such that $\Delta(z, \beta) \in X$.

Using the representation (4) for $\Delta(z, \beta)$ in (10), we obtain

$$
\mu(z, \beta)-\nabla f(z, \beta)=\gamma(z, \beta) \cdot\left[\begin{array}{l}
\xi \\
r
\end{array}\right]
$$

Substituting (5) and (7) into (11), it can be shown after lengthy manipulations that

$$
\begin{aligned}
& \xi_{k+1}=A_{k} \xi_{k}+B_{k} r_{k}, \quad \xi_{0}=0 \\
& -p_{k-1}+A_{k}^{\prime} p_{k}-\bar{x}_{k}=
\end{aligned}
$$




$$
\begin{aligned}
& \beta Q_{k} \xi_{k}+\sum_{i=1}^{m_{c}} \frac{d_{i}}{s_{i}^{2}(z)} a_{k}^{(i)} \\
& B_{k-1}^{\prime} p_{k-1}-\bar{u}_{k}=\beta R_{k} r_{k-1}+\sum_{i=1}^{m_{c}} \frac{d_{i}}{s_{i}^{2}(z)} b_{k}^{(i)} \\
& p_{N}=0
\end{aligned}
$$

Note first that we can rearrange (14) to get

$$
r_{k-1}=\frac{1}{\beta} R_{k}^{-1}\left[B_{k-1}^{\prime} p_{k-1}-\bar{u}_{k}-\sum_{i=1}^{m_{c}} \frac{d_{i}}{s_{i}^{2}(z)} b_{k}^{(i)}\right]
$$

The solution of (12)-(15) is found using a similar procedure to the one used by Faybusovich and Moore (1995). Let

$$
p_{k}=\beta K_{k} \xi_{k}+\rho_{k}, \quad k=0, \cdots, N-1
$$

It can be shown after lengthy manipulations that if $S_{k}$ is the solution of the Riccati equation

$$
\begin{aligned}
& S_{k-1}=Q_{k-1}+A_{k-1}^{\prime} S_{k} A_{k-1} \\
& -A_{k-1} S_{k} B_{k-1}\left[R_{k}+B_{k-1}^{\prime} S_{k} B_{k-1}\right]^{-1} B_{k-1}^{\prime} S_{k} A_{k-1} \\
& S_{N}=Q_{N}
\end{aligned}
$$

then

$$
\begin{aligned}
& K_{k}=-S_{k+1} A_{k} \\
& +S_{k+1} B_{k}\left[R_{k}+B_{k}^{\prime} S_{k+1} B_{k}\right]^{-1} B_{k}^{T} S_{k+1} A_{k} \\
& K_{N}=0 \\
& \xi_{k+1}=\left[A_{k}-B_{k}\left[R_{k}+B_{k}^{\prime} S_{k+1} B_{k}\right]^{-1} B_{k}^{\prime} S_{k+1} A_{k}\right] \xi_{k} \\
& \quad+B_{k} R_{k+1}^{-1} B_{k}^{\prime} \rho_{k}+\zeta_{k} \\
& \xi_{0}=0 \\
& \rho_{k-1}=\alpha_{k}+ \\
& {\left[I-S_{k} B_{k-1}\left[R_{k}+B_{k-1}^{\prime} S_{k} B_{k-1}\right]^{-1} B_{k-1}^{\prime}\right] A_{k}^{\prime} \rho_{k}} \\
& \rho_{N}=0
\end{aligned}
$$

where

$$
\begin{gathered}
\alpha_{k}= \\
{\left[I-S_{k} B_{k-1}\left(R_{k}+B_{k-1}^{\prime} S_{k} B_{k-1}\right)^{-1} B_{k-1}^{\prime}\right]} \\
\times\left[S_{k} \zeta_{k-1}-\bar{x}_{k}-\sum_{i=1}^{m_{c}} \frac{d_{i} a_{k}^{(i)}}{s_{i}^{2}(z)}\right] \\
\zeta_{k}=-B_{k} R_{k+1}^{-1} \bar{u}_{k+1}-B_{k} R_{k+1}^{-1} \sum_{i=1}^{m_{c}} \frac{d_{i} b_{k+1}^{(i)}}{s_{i}^{2}(z)}
\end{gathered}
$$

The solution $\xi_{k}$ of $(20)$ and $r_{k}$ of (16) are

$$
\xi_{k}=\rho_{k}+\sum_{i=1}^{m_{c}} d_{i} \rho_{k}^{(i)}, \quad r_{k}=\eta_{k}+\sum_{i=1}^{m_{c}} d_{i} \eta_{k}^{(i)}
$$

where $\rho_{k}, \rho_{k}^{(i)}$ and $\eta_{k}, \eta_{k}^{(i)}$ are known functions. To calculate $d_{i}$, substitute $\xi$ and $r$ from (24) into (3). We obtain

$$
d_{i}=\sum_{j=1}^{m_{c}} f_{i j} d_{j}+g_{i}, \quad i=1, \cdots, m_{c}
$$

where

$$
\begin{aligned}
f_{i j} & =\sum_{k=1}^{N}\left(a_{k}^{(i)}{ }^{\prime} \rho_{k}^{(j)}+b_{k}^{(i)}{ }^{\prime} \eta_{k-1}^{(j)}\right) \\
g_{i} & =\sum_{k=1}^{N}\left(a_{k}^{(i)}{ }^{\prime} \rho_{k}+b_{k}^{(i)}{ }^{\prime} \eta_{k-1}\right)
\end{aligned}
$$

This is a system of $m_{c}$ linear equations which can be easily solved.

In summary, performance of a Newton step involves:

(a) solving the Riccati equation (18),

(b) solving the linear equations (21) and (20),

(c) solving the $m_{c} \times m_{c}$ linear system (25).

Note that steps (a) and (b) are performed when solving the unconstrained LQ optimal control problem.

For $\lambda=\left(\lambda_{1}, \cdots, \lambda_{m_{c}}\right) \in \mathbf{R}^{m_{c}}$, let $a_{k}(\lambda)$ and $b_{k}(\lambda)$ be be defined by

$$
a_{k}(\lambda)=\sum_{i=1}^{m_{c}} \lambda_{i} a_{k}^{(i)}, \quad b_{k}(\lambda)=\sum_{i=1}^{m_{c}} \lambda_{i} b_{k}^{(i)}
$$

Consider the state-control pair $z^{(1)}=\left(x^{(1)}, u^{(1)}\right)$ which is obtained after performing a Newton step from an arbitrary initial feasible state-control pair $z^{(0)}=\left(x^{0)}, u^{(0)}\right)$, that is $z^{(1)}=z^{(0)}+\Delta\left(z^{(0)}, \beta\right)$. By noting that $u_{k}^{(1)}=$ $u_{k}^{(0)}+r_{k}$, the following result can be derived from (18)(20).

Theorem 2.1 Given any feasible state-control pair $z^{(0)}$ $=\left(x^{(0)}, u^{(0)}\right)$, the implementation of a Newton step $z^{(1)}=z^{(0)}+\Delta\left(z^{(0)}, \beta\right)$ results in the state-control pair $z^{(1)}=\left(x^{(1)}, u^{(1)}\right)$ with control

$$
\begin{aligned}
u_{k}^{(1)} & =-\left(R_{k+1}+B_{k}^{\prime} S_{k+1} B_{k}\right)^{-1} \\
& \times\left[B_{k}^{\prime} S_{k+1} A_{k} x_{k}^{(1)}+B_{k}^{\prime} h_{k+1}(\lambda)+b_{k+1}(\lambda)\right]
\end{aligned}
$$




$$
\begin{aligned}
& \text { where } \lambda^{(1)}=\left(\lambda_{1}^{(1)}, \cdots, \lambda_{m_{c}}^{(1)}\right) \text { with } \\
& \qquad \lambda_{i}^{(1)}=\left(1+\frac{d_{i}}{s_{i}\left(z^{(0)}\right)}\right) \frac{1}{s_{i}\left(z^{(0)}\right)}
\end{aligned}
$$

and

$$
\begin{aligned}
& S_{k-1}=Q_{k-1}+A_{k-1}^{\prime} S_{k} A_{k-1} \\
& -A_{k-1} S_{k} B_{k-1}\left[R_{k}+B_{k-1}^{\prime} S_{k} B_{k-1}\right]^{-1} B_{k-1}^{\prime} S_{k} A_{k-1} \\
& S_{N}=Q_{N} \\
& h_{k}(\lambda)=a_{k}(\lambda)+A_{k}^{\prime} h_{k+1}(\lambda) \\
& -A_{k}^{\prime} S_{k+1} B_{k}\left(R_{k+1}+B_{k}^{\prime} S_{k+1} B_{k}\right)^{-1} \\
& \quad \times\left(B_{k}^{\prime} h_{k+1}(\lambda)+b_{k}(\lambda)\right) \\
& h_{N}(\lambda)=a_{N}(\lambda)
\end{aligned}
$$

Note that $S_{k}$ is independent of $\lambda$ and $\beta$. An immediate consequence of Theorem 2.1 is the following.

Theorem 2.2 The optimal control of the constrained $L Q$ optimal control problem (1)-(3) is of the form

$$
\begin{aligned}
u_{k} & =-\left(R_{k+1}+B_{k}^{\prime} S_{k+1} B_{k}\right)^{-1} \\
& \times\left[B_{k}^{\prime} S_{k+1} A_{k} x_{k}+B_{k}^{\prime} h_{k+1}\left(\lambda^{*}\right)+b_{k+1}\left(\lambda^{*}\right)\right]
\end{aligned}
$$

where $S_{k}$ and $h_{k}\left(\lambda^{*}\right)$ are given by (28)-(29) and $\lambda_{i}^{*} \geq 0$.

We make some important observations. First, if the problem is unconstrained, that is if $a_{k}^{(i)}=b_{k}^{(i)}=0$ and $c_{i}>0$ for $i=1, \cdots, m_{c}$ and $k=1, \cdots, N$, then $h_{k}=$ 0 . It is clear from (26) that the optimal control of the unconstrained LQ optimal control problem is obtained after one Newton step.

Second, the Lagrange multiplier $\lambda^{*}=\left(\lambda_{1}^{*}, \cdots, \lambda_{m_{c}}^{*}\right)$ which yields the optimal control $u^{*}$ can be calculated by solving a sequence of Newton steps and using the expression (27). In this way, the structure of the optimal control is maintained and at the same time, each iterate is feasible.

Finally, the expression (30) for the optimal control $u^{*}$ can also be derived from the Kuhn-Tucker conditions. It follows that $\lambda^{*}$ is the optimal solution of the associated dual problem (an $m_{c}$-dimensional, linearly constrained quadratic optimization problem). However, if we solve the dual problem using a different iterative optimization scheme, each iterate $\hat{\lambda}_{k}\left(\hat{\lambda}_{k} \rightarrow \lambda^{*}\right.$ as $\left.k \rightarrow \infty\right)$ would not give a feasible (sub-optimal) control (a desirable property in many applications). Furthermore, it is known that finite-dimensional interior-point methods are very efficient for problems with many constraints: Few New- ton steps required for a good sub-optimal solution. We expect that the same is true for the infinite-dimensional case (in particular, this is relevant when applied to the continuous-time problem).

\section{CONSTRAINED LQG CONTROL}

The Separation Theorem is a classical result from stochastic optimal control. Though proven in the late 1960's (Wohnam, 1968), it continues to capture the attention of researchers and alternative proofs are still being published (Davis and Zervos, 1995). We now generalize the Separation Theorem to the case where the system is subject to linear inequality constraints. As in Davis and Zervos (1995), we consider the discrete-time linear stochastic system

$$
\begin{aligned}
x_{k+1} & =A_{k} x_{k}+B_{k} u_{k}+w_{k+1}, \quad x_{0} \sim N\left(\mu, P_{0}\right)(31) \\
y_{k} & =C_{k} x_{k}+v_{k}
\end{aligned}
$$

where $x_{0}, w_{1}, \cdots, w_{N}, v_{0}, \cdots, v_{N}$ are independent, zero mean Gaussian random variables such that $E\left[w_{k} w_{l}^{\prime}\right]=$ $\hat{Q}_{k} \delta(k-l), E\left[v_{k} v_{l}^{\prime}\right]=\hat{R}_{k} \delta(k-l)$. The random variables $x_{0}, w_{i}$ and $v_{i}$ are all defined on a probability space $(\Omega, \mathcal{F}, \mathcal{P})$. Let $\mathcal{U}$ be the set of all control processes $u=\left(u_{0}, \cdots, u_{N-1}\right)$ such that $u_{k} \in \mathcal{L}^{2}(\Omega, \mathcal{F}, \mathcal{P})$, and $\mathcal{X}$ the set of all state processes $x=\left(x_{0}, \cdots, x_{N}\right)$ such that $x_{k} \in \mathcal{L}^{2}(\Omega, \mathcal{F}, \mathcal{P})$. The cost and $m_{c}$ constraint functionals are defined on $\mathcal{X} \times \mathcal{U}$ and given by

$$
f_{0}(x, u)=\frac{1}{2} E\left[\sum_{k=1}^{N}\left(x_{k}^{\prime} Q_{k} x_{k}+u_{k-1}^{\prime} R_{k} u_{k-1}\right)\right]
$$

$l_{i}(x, u)=E\left[\sum_{k=1}^{N}\left(a_{k}^{(i)}{ }^{\prime} x_{k}+b_{k}^{(i)}{ }^{\prime} u_{k-1}\right)\right]$

The aim is to find a control process $u=\left(u_{0}, \cdots, u_{N-1}\right)$ from some class of admissible controls which minimizes the cost functional $f_{0}(x, u)$ and satisfies the constraints $l_{i}(x, u) \leq c_{i}\left(i=1, \cdots, m_{c}\right)$. The set of admissible controls are subsets of $\mathcal{U}$.

\subsection{Deterministic full state information case}

This is the problem (1)-(3). Let $u^{*}$ be the optimal control and define

$$
f(\lambda, x, u)=f_{0}(x, u)+\sum_{i=1}^{m_{c}} \lambda_{i} l_{i}(x, u)
$$


If

$$
u(\lambda)=\arg \min _{u}\{f(\lambda, x, u)\}
$$

and

$$
\begin{aligned}
\lambda^{*} & =\arg \max _{\lambda \geq 0}\left[\min _{u}\{f(\lambda, x, u)\}-\lambda^{\prime} c\right] \\
& =\arg \max _{\lambda \geq 0}\left[f(\lambda, x, u(\lambda))-\lambda^{\prime} c\right]
\end{aligned}
$$

then it follows from the Kuhn-Tucker conditions that $u^{*}=u\left(\lambda^{*}\right)$.

Theorem 3.1 Let $\lambda^{*}$ be the optimal solution of

$$
\max _{\lambda \geq 0}\left\{\frac{1}{2} \mu^{\prime} S_{0} \mu+h_{0}^{\prime}(\lambda) \mu+\frac{1}{2} p_{0}(\lambda)-\lambda^{\prime} c\right\}
$$

subject to (28)-(29) and

$$
\begin{aligned}
& p_{k}(\lambda)=p_{k+1}(\lambda)+\left(B_{k}^{\prime} h_{k+1}(\lambda)+b_{k}(\lambda)\right)^{\prime} \\
& \quad \times\left(R_{k+1}+B_{k}^{\prime} S_{k+1} B_{k}\right)^{-1}\left(B_{k}^{\prime} h_{k+1}(\lambda)+b_{k}(\lambda)\right) \\
& p_{N}=0
\end{aligned}
$$

Then $u^{*}=\left(u_{0}^{*}, \cdots, u_{N-1}^{*}\right)$ is the optimal control for the linearly constrained LQ optimal control problem (1)-(3) where

$$
\begin{gathered}
u_{k}^{*}=-\left(R_{k+1}+B_{k}^{\prime} S_{k+1} B_{k}\right)^{-1} \\
\times\left[B_{k}^{\prime} S_{k+1} A_{k} x_{k}+B_{k}^{\prime} h_{k+1}\left(\lambda^{*}\right)+b_{k}\left(\lambda^{*}\right)\right]
\end{gathered}
$$

Remark 3.1 Since (29) and (37) are linear in $\lambda,(36)$ is a quadratic optimization problem over $\lambda$.

\subsection{Stochastic full information case}

In this case, there is full state observation, so $(32)$ is irrelevant. Let $\tilde{\mathcal{F}}_{k} \in \mathcal{F}$ be the $\sigma$-algebra generated by $x_{0}, w_{0}, \cdots, w_{k}$. The class of admissible controls $\tilde{\mathcal{U}}$ is the set of all measurable control processes $u \in \mathcal{U}$ such that $u_{k}$ is measurable with respect to $\tilde{\mathcal{F}}_{k-1}$; that is, $u_{k}$ is a function of at most $x_{0}$ and $\left(w_{0}, \cdots, w_{k-1}\right)$ or equivalently, a function of at most $\left(x_{0}, \cdots, x_{k}\right)$. Let $\tilde{\mathcal{X}}$ be the set of state processes $x$ such that $x_{k}$ is measurable with respect to the $\sigma$-algebra $\tilde{\mathcal{F}}_{k-1}$; that is, $x_{k}$ is a function of at most $x_{0}$ and $\left(w_{0}, \cdots, w_{k-1}\right)$. Then $\tilde{\mathcal{X}} \times \tilde{\mathcal{U}}$ is a vector subspace of $\mathcal{X} \times \mathcal{U}$ and (31) (33)-(34) is a convex optimization problem on a linear variety of $\tilde{\mathcal{X}} \times \tilde{\mathcal{U}}$. Furthermore, the Kuhn-Tucker conditions are necessary and sufficient for optimality. Let $f(\lambda, x, u)$ be defined by $(35)$ with $f_{0}(x, u)$ and $l_{i}(x, u)$ given by $(33)-(34)$ respectively. Once again, it follows from the Kuhn-Tucker conditions that if

$$
\begin{aligned}
u(\lambda) & =\arg \min _{u \in \tilde{\mathcal{U}}}\{f(\lambda, x, u)\} \\
\lambda^{*} & =\arg \max _{\lambda \geq 0}\left[\min _{u \in \tilde{\mathcal{U}}}\{f(\lambda, x, u)\}-\lambda^{\prime} c\right] \\
& =\arg \max _{\lambda \geq 0}\left[f(\lambda, x, u(\lambda))-\lambda^{\prime} c\right]
\end{aligned}
$$

then $u^{*}=u\left(\lambda^{*}\right)$ is the optimal control. The Separation Theorem for the full information constrained LQG problem can be stated as follows.

Theorem 3.2 Let $\lambda^{*}$ be the optimal solution of the dual problem stated in Theorem 3.1. Then

$$
\begin{gathered}
u_{k}^{*}=-\left(R_{k+1}+B_{k}^{\prime} S_{k+1} B_{k}\right)^{-1} \\
\times\left[B_{k}^{\prime} S_{k+1} A_{k} x_{k}+B_{k}^{\prime} h_{k+1}\left(\lambda^{*}\right)+b_{k}\left(\lambda^{*}\right)\right]
\end{gathered}
$$

is the optimal control for the full information linearly constrained LQG optimal control problem (31) (33)(34).

\section{Proof:}

For any given $\lambda \geq 0$, it can be shown using dynamic programming that

$$
\begin{aligned}
& \min _{u \in \tilde{\mathcal{U}}}\{f(\lambda, u)\}=\frac{1}{2} \mu^{\prime} S_{0} \mu+h_{0}^{\prime}(\lambda) \mu+\frac{1}{2} p_{0}(\lambda) \\
& \quad+\frac{1}{2} \sum_{n=1}^{N} \operatorname{tr}\left\{\hat{Q}_{k} S_{k}\right\}
\end{aligned}
$$

The result follows from the Kuhn-Tucker conditions and the fact that $\operatorname{tr}\left\{\hat{Q}_{k} S_{k}\right\}$ is independent of $\lambda$.

Remark 3.2 If $\lambda$ results in a feasible control of the form (38) for the deterministic constrained LQ problem, then the control (39) will also be feasible. Therefore, the full information problem can be solved by solving the deterministic LQ problem using the IPM result in Section 2 and (27) with all the desirable characteristics associated with IPM's (fast convergence and feasible sub-optimal solutions) still being true.

\subsection{Stochastic partial information case}

This is the problem (31)-(34). Let $\overline{\mathcal{F}}_{k}$ be the $\sigma$-algebra generated by the random variables $y_{0}, \cdots, y_{k}$. The class of admissible controls $\overline{\mathcal{U}}$ is the set of control processes $u \in \mathcal{U}$ such that $u_{k}$ is measurable with respect to the $\sigma$-algebra $\overline{\mathcal{F}}_{k-1}$; that is, $u_{k}$ is a function of at most $\left(y_{0}, \cdots, y_{k-1}\right)$.

Let $\hat{x}_{k \mid k-1}=E\left[x_{k} \mid y_{0}, \cdots, y_{k-1}\right]=E\left[x_{k} \mid \overline{\mathcal{F}}_{k-1}\right]$. It is a well known from estimation theory (Anderson and 
Moore, 1979) that $\hat{x}_{k \mid k-1}$ is given by the Kalman filter

$\hat{x}_{k+1 \mid k}=A_{k} \hat{x}_{k \mid k-1}+B_{k} u_{k}-\hat{K}_{k} \nu_{k}, \quad \hat{x}_{0 \mid-1}=\mu$

where the Kalman gain $\hat{K}$ is

$$
\begin{aligned}
& \hat{K}_{k}=A_{k} \Sigma_{k \mid k-1} C_{k}^{\prime}\left(\hat{R}_{k}+C_{k} \Sigma_{k \mid k-1} C_{k}^{\prime}\right)^{-1} \\
& \Sigma_{k \mid k-1}=E\left[\left(x_{k}-\hat{x}_{k \mid k-1}\right)\left(x_{k}-\hat{x}_{k \mid k-1}\right)^{\prime}\right] \text { is given by } \\
& \quad \Sigma_{k+1 \mid k}=\hat{Q}_{k}+A_{k} \Sigma_{k \mid k-1} A_{k}^{\prime} \\
& \quad+A_{k} \Sigma_{k \mid k-1} C_{k}^{\prime}\left[\hat{R}_{k}+C_{k} \Sigma_{k \mid k-1} C_{k}^{\prime}\right]^{-1} C_{k} \Sigma_{k \mid k-1} A_{k}^{\prime} \\
& \Sigma_{0 \mid-1}=P_{0}
\end{aligned}
$$

and innovations process $\nu_{k}=y_{k}-C_{k} \hat{x}_{k \mid k-1} \in \overline{\mathcal{F}}_{k}$. We state the following without verification. The reader may refer to Anderson and Moore (1979) for precise details.

$$
E\left[\left(x_{k}-\hat{x}_{k \mid k-1}\right) \hat{x}_{k \mid k-1}^{\prime}\right]=0 .
$$

Second, the innovations process is a white noise process satisfying

$$
E\left[\nu_{k}\right]=0, E\left[\nu_{k} \hat{x}_{k \mid k-1}^{\prime}\right]=0, E\left[\nu_{k} \nu_{l}^{\prime}\right]=\hat{G}_{k} \delta(k-l)
$$

It follows that

$$
\begin{aligned}
f_{0}(x, u)=\frac{1}{2} & E\left[\sum_{k=1}^{N}\left(\hat{x}_{k \mid k-1}^{\prime} Q_{k} \hat{x}_{k \mid k-1}+u_{k-1}^{\prime} R_{k} u_{k-1}\right)\right] \\
& +\frac{1}{2} \sum_{k=0}^{N} \operatorname{tr}\left\{Q_{k} \Sigma_{k \mid k-1}\right\}
\end{aligned}
$$

Note $\Sigma_{k \mid k-1}$ is deterministic and independent of $u$. Since it is true that $E\left[x_{k}\right]=E\left[E\left[x_{k} \mid \overline{\mathcal{F}}_{k-1}\right]\right]=E\left[\hat{x}_{k \mid k-1}\right]$, the constraint functional (34) becomes

$$
l_{i}(x, u)=E\left[\sum_{k=1}^{N}\left(a_{k}^{(i)}{ }^{\prime} \hat{x}_{k \mid k-1}+b_{k}^{(i)}{ }^{\prime} u_{k-1}\right)\right]
$$

Therefore, the partial information problem (31)-(34) is equivalent to the full information problem (40)-(42). The Separation Theorem for the partial information LQG problem follows from Theorem 3.2.

Theorem 3.9 Let $\lambda^{*}$ be the optimal solution of the dual problem stated in Theorem 3.1. Then

$$
\begin{gathered}
u_{k}^{*}=-\left(R_{k+1}+B_{k}^{\prime} S_{k+1} B_{k}\right)^{-1} \\
\times\left[B_{k}^{\prime} S_{k+1} A_{k} \hat{x}_{k \mid k-1}+B_{k}^{\prime} h_{k+1}\left(\lambda^{*}\right)+b_{k}\left(\lambda^{*}\right)\right]
\end{gathered}
$$

is the optimal control for the linearly constrained LQG optimal control problem (31)-(34) where the conditioned mean estimate $\hat{x}_{k \mid k-1}$ is the output of the Kalman filter (40).

Remark 3.3 Once again, if $\lambda$ results in a feasible control of the form (38) for the deterministic LQ problem, it also gives a feasible control of the form (43) for the partial information LQG problem. The partial information LQG problem can therefore be solved using the IPM results from Section 2 (see Remark 3.2).

\section{Conclusion}

Based on the results obtained by Faybusovich and Moore (1995) we have shown that the linearly constrained LQ optimal control problem can be solved by solving a sequence of unconstrained LQ problems together with a system of finite-dimensional linear algebraic equations. We have also proven the Separation Theorem for the linearly constrained LQG problem and shown that the IPM result for the constrained LQ problem can be used to calculate the optimal control for the constrained LQG problem, while preserving favourable properties associated with IPM's (fast convergence and feasible sub-optimal iterates).

\section{REFERENCES}

Anderson, B. D. O. and J. B. Moore (1979). Optimal Filtering. Prentice-Hall.

Anderson, B. D. O. and J. B. Moore (1989). Optimal Control: Linear Quadratic Methods. Prentice-Hall.

Davis, M. H. A. and M. Zervos (1995). A new proof of the discrete-time lqg optimal control theorems. IEEE Transactions on Automatic Control 18(1), 1-13.

den Hertog, D. (1994). Interior Point Approach to Linear, Quadratic and Convex Programming. Kluwer Academic Publishers.

Faybusovich, L. (1982). Application of a reduction method to thcase analysis of linear dynamical systems with phase constraints. Automation and Remote Control 8, 1014-1020.

Faybusovich, L. and J. B. Moore (1995). A long-step path-following algorithm for the convex quadratic programming problem in a hilbert space. Proc. 34 th IEEE Conference on Decision and Control.

Faybusovich, L. and J. B. Moore (1996). Infinite dimensional quadratic optimization: interior-point methods and control applications. Journal of Applied Mathematics and Optimization (to appear).

Wohnam, W. M. (1968). On the separation theorem of stochastic control. SIAM J. Control 6, 312-326. 